\title{
The New Era of Virtual Reality Locomotion: A Systematic Literature Review of Techniques and a Proposed Typology
}

\author{
Costas Boletsis \\ SINTEF Digital, Forskningsveien 1, 0373 Oslo, Norway; konstantinos.boletsis@sintef.no
}

Received: 7 September 2017; Accepted: 25 September 2017 ; Published: 28 September 2017

\begin{abstract}
The latest technical and interaction advancements that took place in the Virtual Reality (VR) field have marked a new era, not only for VR, but also for VR locomotion. Although the latest advancements in VR locomotion have raised the interest of both researchers and users in analyzing and experiencing current VR locomotion techniques, the field of research on VR locomotion, in its new era, is still uncharted. In this work, VR locomotion is explored through a systematic literature review investigating empirical studies of VR locomotion techniques from 2014-2017. The review analyzes the VR locomotion techniques that have been studied, their interaction-related characteristics and the research topics that were addressed in these studies. Thirty-six articles were identified as relevant to the literature review, and the analysis of the articles resulted in 73 instances of $11 \mathrm{VR}$ locomotion techniques, such as real-walking, walking-in-place, point and teleport, joystick-based locomotion, and more. Results showed that since the VR revival, the focus of VR locomotion research has been on VR technology and various technological aspects, overshadowing the investigation of user experience. From an interaction perspective, the majority of the utilized and studied VR locomotion techniques were found to be based on physical interaction, exploiting physical motion cues for navigation in VR environments. A significant contribution of the literature review lies in the proposed typology for VR locomotion, introducing four distinct VR locomotion types: motion-based, room scale-based, controller-based and teleportation-based locomotion.
\end{abstract}

Keywords: human-computer interaction; literature review; locomotion; typology; virtual reality

\section{Introduction}

Over the last few years, Virtual Reality (VR) has undergone a major hardware-driven revival, which has had significant effects on the ways users experience and use VR [1,2]. The introduction of the Oculus Rift Development Kit 1 in 2013 is considered a significant milestone for VR, indicating when the VR revival took place and when VR became accessible, up-to-date and relevant again [1,3-5]. The low acquisition cost of VR hardware transformed VR into a widely-accessible and popular technology. At the same time, the quality of virtual environments increased rapidly, offering realistic graphics and full immersion, while surpassing the lack of intuitive multi-user capabilities of the past, and pushing the boundaries of next-generation social platforms [6-8]. From a Human-Computer Interaction (HCI) perspective, the technological revival of VR has produced new and updated interaction metaphors, designs and tools, thus affecting the resulting user experiences and the research of the field [3]. Overall, the VR revival marked what has been characterized as the "new era of Virtual Reality" [9-11].

VR locomotion, an important interaction component that enables navigation in VR environments, was heavily affected by the technological change [12,13]. Since the early days of VR, various locomotion techniques have been developed and studied, targeting seamless and user-friendly navigation in virtual environments $[12,14]$, while key theoretical models and classifications were developed to ground the constructive contributions of VR locomotion techniques, such as the taxonomies of 
Bowman et al. [15,16] and Arns [17]. The latest technical and interaction advancements of VR marked a new era for VR locomotion, as well. As a result, new VR locomotion techniques and related elements have been developed, and past ones have been significantly updated. For instance, point-and-click teleportation is now a mainstream VR locomotion technique, fully supported by commercial Head-Mounted Displays (HMD), such as the HTC Vive and the Oculus Rift [12], while motion-based locomotion techniques, including swimming, climbing, flying and walking-in-place, have become more robust and user-friendly [18,19]. Joystick-based locomotion now addresses virtual sickness with effective dynamic field-of-view adjustments (blinders) [20], and the real-walking locomotion technique, which was a cumbersome construction for the lab [21], now comes out-of-the-box with commercial headsets [22,23]. Although the latest advancements in VR locomotion have raised the interest of both researchers and users (e.g., [24,25]), in terms of understanding, developing, utilizing and experiencing the VR locomotion techniques, the field of research on VR locomotion, in its new era, is still uncharted.

In this work, the focus is placed on the new era of VR locomotion by exploring the VR locomotion techniques that have been studied since the VR revival milestone. To do so, a systematic literature review is conducted, investigating empirical studies of HMD-based VR locomotion techniques from 2014-2017. The aim of the review is to (1) organize and map the VR locomotion research field in its current form; (2) identify research gaps in the field that warrant further exploration; and (3) construct new conceptual knowledge that provides the theoretical grounding for future VR locomotion-related empirical and constructive work. The ultimate intention of this work is for VR researchers, developers and users to have an overview of the current state-of-the-art techniques in the VR locomotion research field, to be able to make sense of these techniques and, ultimately, to ground their future contributions in the herein synthesized theoretical knowledge.

The paper is organized as follows. First, the literature review process is described, followed by an overview of the reviewed papers (Section 2 and Table 1). Next, the findings from the review process are presented (Section 3). Finally, a discussion of the key findings and recommendations for future research are presented, along with implications for the research (Section 4).

\section{Method}

This study has been undertaken as a systematic literature review based on the original guidelines, as proposed by Kitchenham [26] and Brereton et al. [27] and implemented by Beecham et al. [28]. In accordance with the guidelines, the following steps were taken:

1. identify the need for a systematic literature review,

2. formulate the research questions of the review,

3. carry out a comprehensive, exhaustive search for primary studies,

4. assess and record the quality of included studies,

5. classify the data needed to answer the research questions,

6. extract data from each included study,

7. summarize and synthesize the study results (meta-analysis),

8. interpret the results to determine their applicability,

9. write-up the study as a report.

To ensure that no important material was overlooked, additional searches of key conference proceedings, journals and authors were performed directly. Furthermore, secondary searches based on references found in our primary studies were conducted.

\subsection{Research Questions}

To assess the current state of the research in the VR locomotion field, the literature review will address three research questions. Since the VR revival:

- $\quad$ RQ1: Which VR locomotion techniques have been studied? 
- RQ2: Which are the interaction-related characteristics of the studied VR locomotion techniques?

- RQ3: Which VR locomotion-related research topics are being addressed in the reviewed studies?

RQ1 is focusing on identifying the VR locomotion techniques that are directly studied or the techniques whose elements are studied. To explore further and analyze the retrieved techniques, their interaction aspects are important, and RQ2 examines those aspects. RQ1 and RQ2 focus on the interaction of the reviewed techniques, while RQ3 examines the research on these techniques and the topics it addresses.

\title{
2.2. Search Strategy
}

A systematic search of the literature was performed in the Scopus academic search engine. For the examined subject, the ACMand IEEE academic databases were considered the most relevant, due to their focus on HCI issues and technical aspects, respectively. The Scopus engine searches through the ACM and IEEE databases, along with the databases of other publishers, such as Elsevier, Springer, Taylor \& Francis, Sage, Emerald, Oxford University Press, Cambridge University Press and many more. Scopus was chosen from among other academic search engines (e.g., Google Scholar, Web of Science) for the main search process due to its wider coverage of related journals, its flexible result-filtering capabilities and the consistent accuracy of its results $[29,30]$.

The search was focused on VR locomotion techniques, as supported by empirical studies, published between the first complete publication year after the VR revival, i.e., January 2014, and the date of this search, i.e., June 2017. The publications' abstracts were utilized for the retrieval of relevant articles, utilizing the following Scopus database advanced-search query string:

\begin{abstract}
ABS (("locomotion" OR "navigation technique") AND ("empirical" OR "studied" OR "study" OR "evaluation" OR "evaluate" OR "examination" OR "examine") AND ("virtual reality" OR "virtual environment" OR "virtual world")) AND (LIMIT-TO (PUBYEAR,2017) OR LIMIT-TO (PUBYEAR, 2016) OR LIMIT-TO (PUBYEAR, 2015) OR LIMIT-TO (PUBYEAR, 2014)).
\end{abstract}

Finally, applicable articles were also identified through backward reference searching, i.e., by screening the reference lists of the retrieved publications [31]. Scopus, Google Scholar and Web of Science were utilized for the backward reference searching to run general searches of specific references and to identify relevant articles.

\subsection{Inclusion and Exclusion Criteria}

Peer-reviewed articles with the following characteristics, published between January 2014 and June 2017, were included:

- written in English,

- including at least one VR locomotion technique,

- including a user study that examines direct or indirect aspects of the VR locomotion technique(s),

- having a fully-immersive VR setup, utilizing HMDs.

The peer-review process adds to the credibility and reliability of the publications. The evaluation of VR locomotion techniques through user studies was considered a significant criterion to present existing and usable systems beyond the conceptual level. HMD-based, fully-immersive VR was chosen as the appropriate setup so that the results of the literature review are technologically up-to-date and, at the same time, are of significance for researchers and for regular users, who now have access to these low to medium cost solutions. 
Consequently, articles with the following characteristics were excluded:

- utilizing exclusively projection-based, desktop-based or tablet-based virtual environments,

- addressing solely conceptual matters of VR locomotion (theoretical models, frameworks, literature reviews, etc.),

- not including an empirical, user study,

- utilizing VR locomotion techniques as a technological/research tool for studying a different, unrelated topic.

\subsection{Screening Process and Results}

The screening process and its results are visualized in Figure 1.

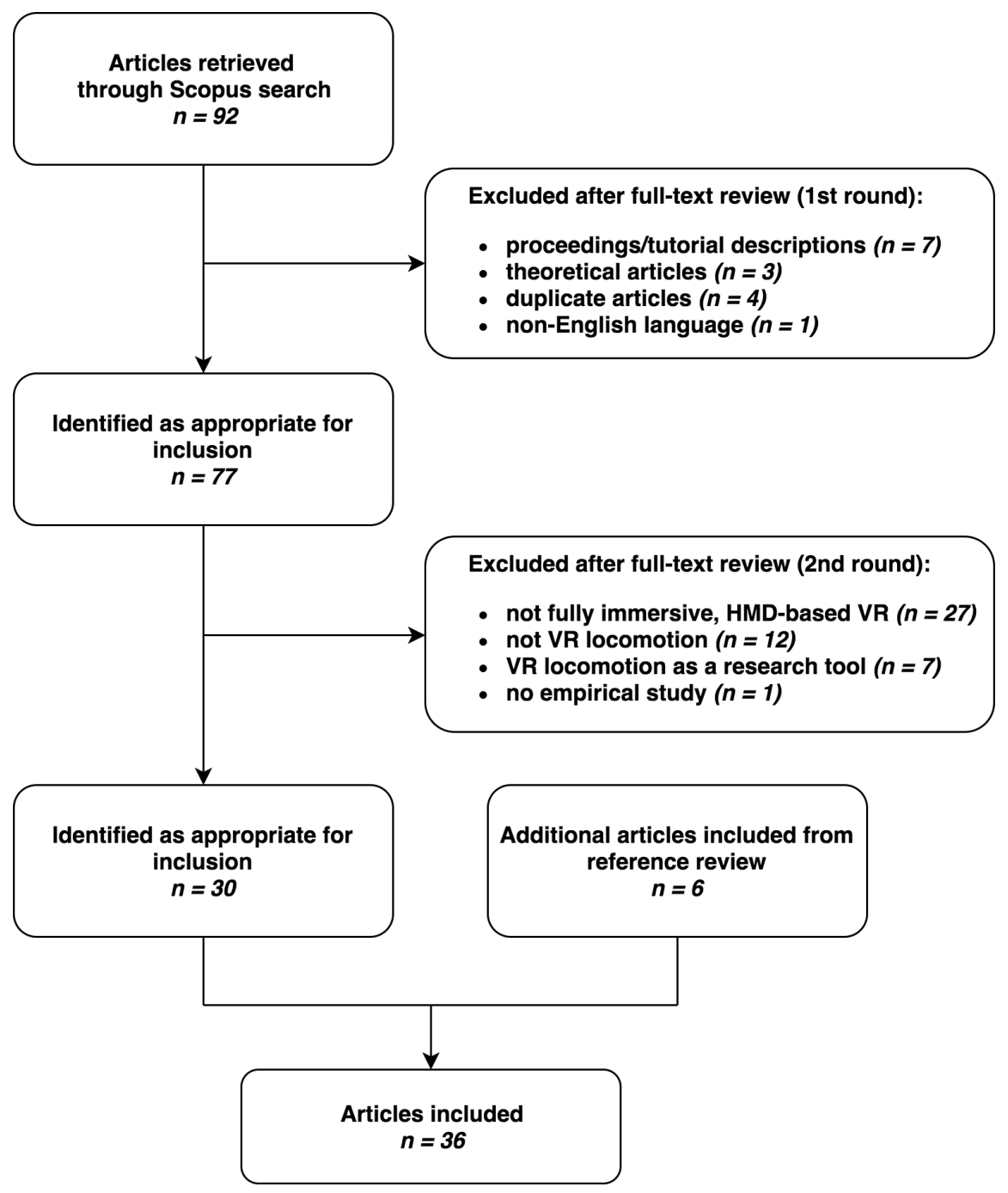

Figure 1. Flowchart of included/excluded articles. HMD, Head-Mounted Display.

The initial search elicited 92 articles (which can be retrieved at www.boletsis.net/mti2017/scopusresults.pdf), and the initial screening of studies was based on their full text, excluding noticeably irrelevant studies. In total, 77 articles were identified as appropriate for inclusion, and they were moved to the second screening round.

For the second round of screening, the full text of the articles identified as appropriate for inclusion $(n=77)$ was again retrieved and reviewed. The author, along with an independent expert of the field, examined a sample of 20 randomly-selected papers from the pool of extracted articles. A $95 \%$ 
inter-rater agreement on the inclusion/exclusion decision was recorded between the two reviewers. Disagreements were discussed, and a decision was made. This high level of agreement provided considerable confidence in the inclusion/exclusion decisions.

In total, 30 articles were identified as appropriate for inclusion after the second screening round. Then, backward reference searching of the extracted articles' references took place, resulting in six articles that fulfilled the inclusion criteria.

Finally, 36 articles were identified as relevant to the current review. The author and the independent expert reviewed all 36 articles independently. The categories and themes of the review were conjointly shaped by the two reviewers, based on the data extraction process. The final validation exercise of the review demonstrated a high level of agreement between the author and the independent expert $(88.8 \%)$, and disagreements were discussed and settled.

\subsection{Data Collection}

The screening process resulted in 36 articles that satisfied the inclusion criteria. The data extracted from each article were:

- the source and full reference,

- the description and title of the VR locomotion technique(s),

- the interaction aspects of the VR locomotion technique(s) (e.g., interaction type, movement type, VR interaction space, devices, etc.),

- the research topic of the empirical study.

If data were missing, the study authors were contacted. The two reviewers, i.e., the author and the independent expert, jointly performed the data extraction process.

\subsection{Data Analysis}

The collected data were synthesized by identifying themes emanating from information reported in each accepted paper and related to the research questions. The themes were classified into a concept matrix to facilitate comparisons. A concept matrix makes the transition from an author- to concept-centric literature review, provides structure and helps in clarifying the concepts of the review for the reader [32]. Table 1 shows the concept matrix of the literature review.

The main themes identified in the review and tabulated were:

- the VR locomotion techniques (addressing RQ1),

- the interaction aspects of the techniques (addressing RQ2),

- the research topics of the empirical studies (addressing RQ3).

The identification of the VR locomotion techniques, their interaction aspects and the empirical studies' research topics were based on the description provided in the articles, as crosschecked with other related and/or reviewed publications in the field to establish their scientific soundness, mainly towards nomenclature and interaction features. Then, the identified themes were normalized and classified so they would be easily comparable and so they would fit into the concept matrix in a valid and lossless way. Comparative studies that included two or more locomotion techniques were tabulated in a respective number of rows. 
Table 1. The literature review.

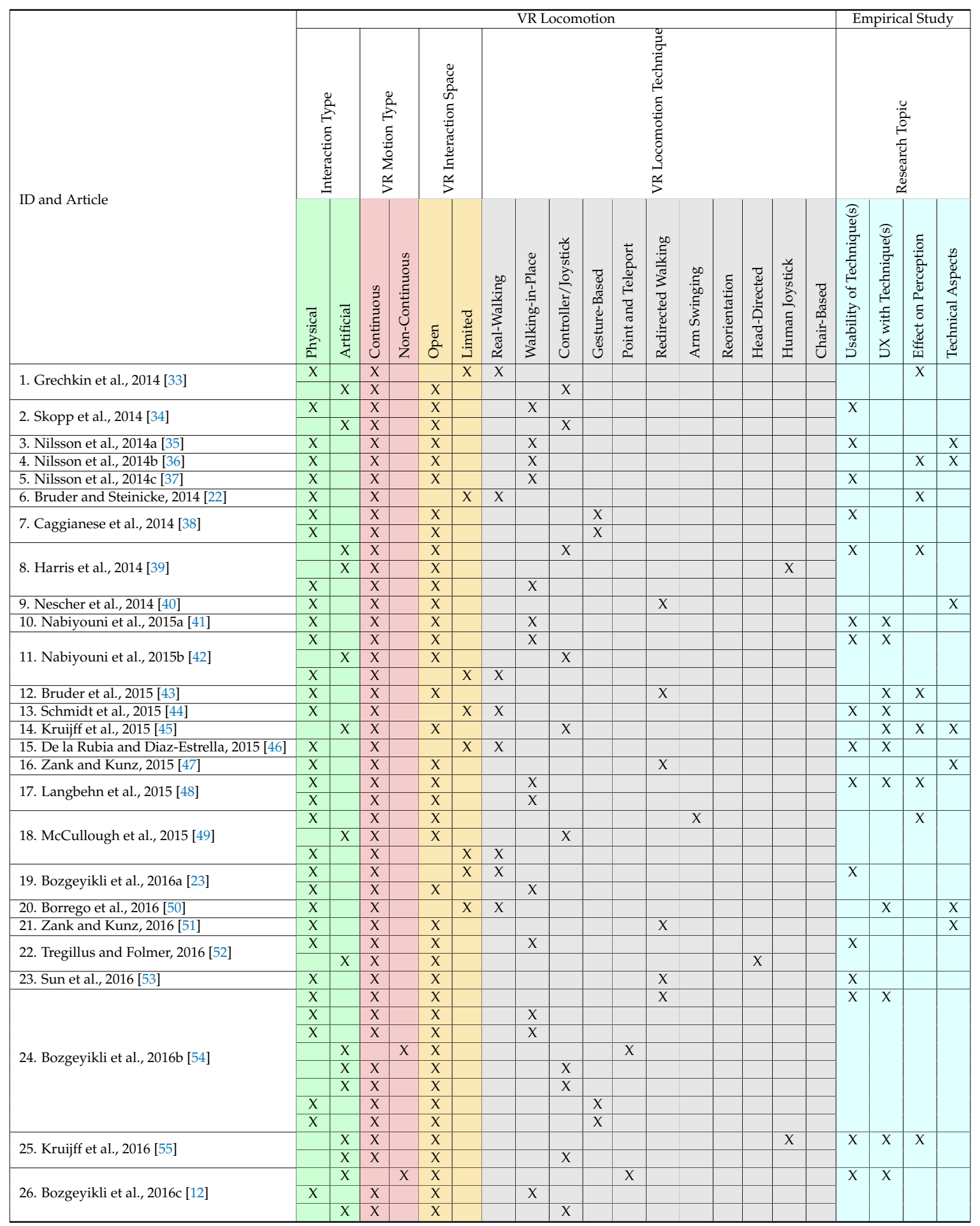


Table 1. Cont.

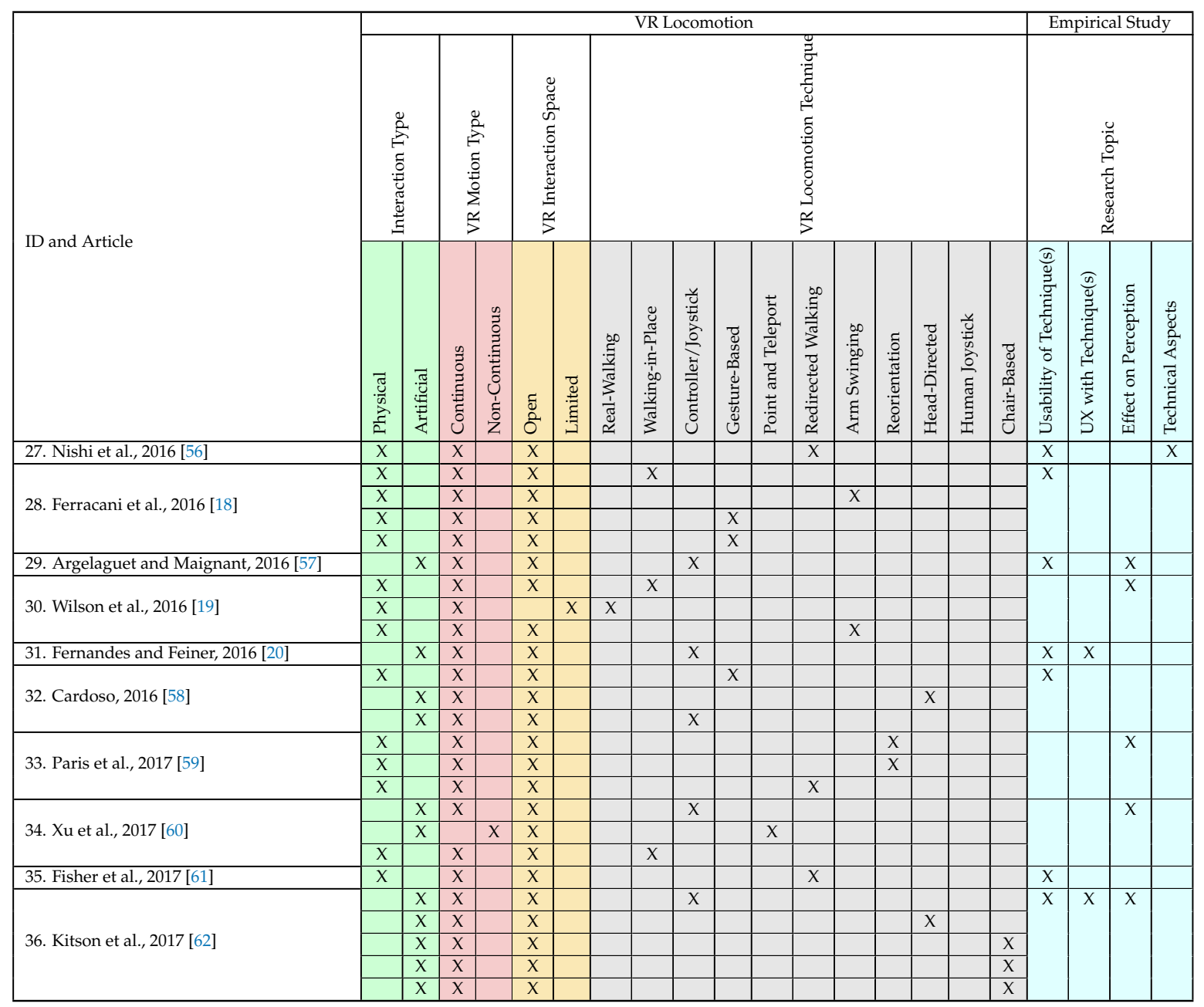

\section{Results}

\subsection{VR Locomotion Techniques}

The literature review documented 73 instances of 11 locomotion techniques in the 36 reviewed empirical studies. The main features of the 11 documented VR locomotion techniques are presented as follows.

- Real-walking: The user walks freely inside a limited physical space. The user's position and orientation are determined, usually by tracking the HMD's position [22,33] or the user's limb movements [44,46,50].

- Walking-in-place: The user performs virtual locomotion by walking in place, i.e., using step-like movements while remaining stationary. The user's limb movements can be tracked, or stepping and treadmill-like input devices, such as the Stepper Machine [54] and VirtuSphere [34], can be used to register the step-like movements and translate them into VR motion [48,52].

- Controller/joystick: The user uses a controller to direct the movement in the virtual environment. The kind of controller can range from a simple joystick [42,54] to a game controller [20,34,42], a keyboard [57] or a trackball [54].

- Gesture-based: The user makes gestures to direct virtual movement. The various gestures (such as tap [18], push [18] and flying [54]) are tracked by input devices, such as the Leap Motion or Microsoft Kinect, and translated into VR motion [18,54,58]. 
- Teleportation: The user points to where he/she wants to be in the virtual world, and the virtual viewpoint is instantaneously teleported to that position. The visual "jumps" of teleportation result in virtual motion being non-continuous [54]. The pointing can take place by using a controller [60] or making a pointing gesture [12,54].

- Redirected walking: The user walks freely inside a limited physical space, while being able to explore unlimited virtual environments by employing so-called redirection techniques. These techniques try to introduce an unnoticeable mismatch between the user's real and virtual movements to compress the larger virtual environment into a limited tracking space [40,47].

- Arm swinging: Users swing their arms while remaining stationary, and their arm movements are translated into VR motion $[18,19,49]$. The arm movements can be tracked by body-tracking devices (e.g., Microsoft Kinect [18]) or wearable and held devices (e.g., armbands and controllers [19,49]).

- Reorientation: The user walks freely inside a limited physical space, while being able to explore unlimited virtual environments by employing so-called reorientation. The reorientation is achieved by modifying the rotational gain of the users, so they physically turn around when they meet the boundaries of the physical space, thus allowing for continued travel in both worlds [59].

- Head-directed: The user uses head movements of the HMD to control movement. The VR motion speed can be fixed or it can be controlled by the forward/backward pitching of the user's head $[52,58,62]$.

- Human joystick: The user stands and leans on a sensing board (e.g., Wii balance board) to produce forward, backward and sideways (strafing) motions, as well as turning during forward motion [39,55].

- Chair-based: The user sits on a stool chair, which acts as an input device, and the stool rotation and tilt are translated into VR forward/backward and turning motions [62]. The technique can have various implementations (such as NaviChair, MuvMan, Swivel Chair [62]).

The walking-in-place locomotion technique was the most utilized (17 instances), followed by the controller/joystick-enabled locomotion technique (15 instances). The 11 locomotion techniques and their number of instances, as documented from the 36 reviewed articles, are visualized in Figure 2. These results answer RQ1.

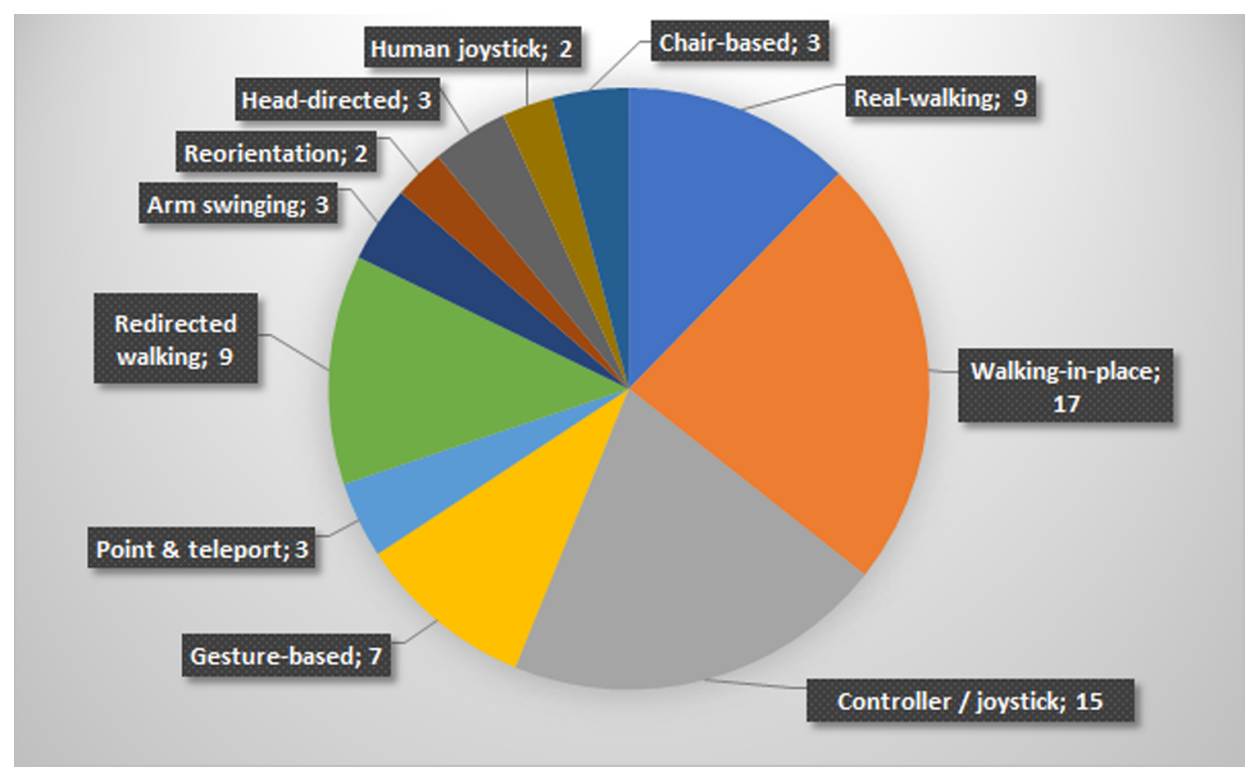

Figure 2. The 11 locomotion techniques and their number of instances, as documented from the 36 reviewed articles. 


\subsection{Interaction Aspects}

To address RQ2, the interaction aspects of the reviewed locomotion techniques were extracted. The resulted interaction-related themes were: the interaction type, the VR motion type and the VR interaction space.

The interaction type of the VR locomotion technique describes the way in which the user triggers VR navigation. Therefore, locomotion can be physical, i.e., exploiting physical motion cues for navigation and translating natural movement to VR motion through some kind of body tracking, or it can be artificial, i.e., utilizing input devices to direct VR motion and navigation [63]. The literature review documented that 47 out of the 73 reviewed locomotion techniques implemented physical interaction, whereas 26 were artificial.

The VR motion type assesses the nature of the user's motions in the VR environment. It can be characterized as continuous, supporting smooth, uninterrupted movement in the virtual environment, or non-continuous, providing instantaneous, non-continuous movement transitions [57]. The majority of the reviewed locomotion techniques $(n=68)$ implemented continuous VR motion, with only five having implemented non-continuous motion.

Finally, VR locomotion techniques can operate in an open VR interaction space, supporting navigation in a virtual environment that surpasses the limits of the real environment, or they can provide limited interaction space capabilities due to the limitations that the physical environment places on the size of the virtual one [12]. The majority of the reviewed locomotion techniques supported open VR interaction spaces $(n=64)$, and nine of them supported limited ones.

\subsection{Research Topics}

To address RQ3, the research topics of the reviewed studies were extracted, resulting in the main themes of usability, User Experience (UX), user perception and technical aspects.

Usability refers to the ease of use of the VR locomotion technique by the users, while UX represents "a turn to experience" [64], focusing on the experience of the users with the VR locomotion technique [65]. The effect of the VR locomotion technique on user perception is also a documented topic that relates to physiological responses while using the VR locomotion technique and that addresses such issues as object location memory, as well as motion, distance, time and speed perception in VR environments [66]. Technical aspects refer to pure technical performances and objective measurements from user sessions with the VR locomotion technique.

Of the 73 reviewed studies, 23 studied the usability of the techniques, 13 studied the UX with the techniques, 14 focused on the effect of the VR locomotion techniques on user perception and eight studied the technical performances of the techniques. Naturally, more than one topic could be addressed in each study, leading to interesting observations that are analyzed further in Section 4.

\section{Discussion}

In the past, the field of VR locomotion has been quite diverse, with various hardware, software, and environments combined to form VR locomotion techniques [15]. This diversity made the conceptual contributions of the research field challenging, possibly because it was extremely difficult for researchers to find a common ground on which to compare or review VR locomotion techniques under an "umbrella" concept. That could be the reason there has not yet been a general VR locomotion literature review. In general, the current systematic literature review showed that the VR revival and the devices that were introduced recently offered a level of technical homogeneity for VR locomotion techniques, thus providing a common ground and allowing for the comparison and analysis of these techniques. Naturally, during the course of this review, a number of challenging issues had to be dealt with (described in Section 4.4) to establish that the reviewed studies could be utilized for meaningful results, while respecting and emphasizing the interaction-oriented nature of the review. Nevertheless, the author's general feeling following this review is that the new era of VR locomotion 
finds the field mature and homogeneous enough for researchers to map it and to develop further significant conceptual knowledge for the research community and the public.

\subsection{VR Locomotion Techniques and Interaction Aspects}

Several observations can be made from the reviewed and studied VR locomotion techniques.

First, walking-in-place and controller/joystick-based locomotion are the most studied techniques, and they are are the main representatives of two different interaction types: physical vs. artificial. Considering the sum of the reviewed locomotion techniques, physical interaction in VR locomotion is much more utilized and studied, as it can trigger intuitive user responses, and it may not add an extra cognitive load with motion instructions. On the other hand, artificial interaction allows for a less physically-intense experience, with the user being stationary and simply using a controller; however, it can be cognitively intense, and it can lead more easily to VR sickness [20]. That may well be the reason that artificial interaction is less utilized in the reviewed studies. The literature review also showed an overwhelming preference for continuous VR motion in open VR environments, which could be explained by the fact that those conditions allow for a consistent VR presence, unconstrained by visual disruptions (e.g., visual "jumps") or space size limitations.

Another important observation is that the VR teleportation technique is not studied or utilized as much. Bearing in mind that teleportation is one of the dominant VR locomotion techniques utilized in many VR applications and games and that it comes out-of-the-box with current commercial systems, such as the HTC Vive and the Oculus Rift, one would expect more empirical studies utilizing and studying the technique. However, this is not the case, and this represents a discovered gap that can be addressed by future studies on VR locomotion.

Finally, even though the resulting interaction-related themes of the review (Section 3.2) concern the new era of VR locomotion, similarities can be drawn between them and the previously established conceptual work of the field. Bowman et al.'s taxonomy [15] introduced the components of virtual traveling (direction, velocity and input conditions). The VR motion type shares common characteristics with the component of input conditions, i.e., the way in which the technique specifies VR motion. Moreover, in an expanded version of Bowman et al.'s framework [16], the size of the environment was considered significant for the performance of the VR locomotion technique, a characteristic represented in the theme of VR interaction space. Arns' work [17] on extending Bowman et al.'s taxonomy and introducing the concept of physical and virtual interaction regarding the rotation of the virtual environment and the translation of the user's viewpoint presents common elements with the interaction type theme. Naturally, the resulting interaction-related themes only share common roots with the conceptual works mentioned above, not only because of their different methodological approaches (with the current work being a literature review and those being taxonomies and frameworks), but also because those works took place decades ago; since then, VR locomotion has changed and advanced significantly. Nevertheless, the interaction core of VR locomotion has remained, and three significant elements were identified in this study, paving the way for further conceptual contributions (Section 4.3).

\subsection{Research Topics}

The review of research topics revealed a strong focus on usability when it comes to VR locomotion. However, the study of user experience and user perception when navigating VR environments seems overlooked. In general, the results show a system-centric instead of a user-centric research approach when it comes to the study of VR locomotion issues. The research topics of usability and technical aspects can be considered to offer a system-centric research approach, examining the VR locomotion technology itself, while user experience and user perception to provide a user-centric research approach, investigating how users experience the VR locomotion systems. Under this categorization, 14 studies adopted a system-centric research approach; seven studies were user-centric; and 15 followed a mixed approach, combining both. Most of the studies were mixed, focusing their research on both the technology and the user of the VR locomotion system. However, when it comes to pure system-centric 
vs. user-centric approaches, it is obvious that the main research focus is on the VR locomotion system and technology, overshadowing the VR locomotion user. From this review, one could conclude that the technology surrounding VR locomotion techniques is adequately studied, and it has matured over these years. As a future suggestion, an outside-in approach could be adopted, shifting the focus from the technology to the user and empirically studying his/her experience with VR locomotion even further.

Another research-related observation has to do with the number of comparative, empirical studies that were reviewed. Half of the reviewed empirical studies (18 of 36) were comparative. Judging by the number and examining it in terms of what it means for the field, there could be a "glass being half-empty or half-full" kind of argument. However, if this observation is combined with the need for more user-centric research, then a future increase in the number of comparative, empirical studies would potentially strengthen the field, allowing researchers to investigate several different forms of VR locomotion and their effect on users at the same time.

\subsection{VR Locomotion Typology}

The results of the literature review allowed for the classification of the VR locomotion techniques, which are supported by empirical studies that have been conducted since the VR revival. The documentation of the techniques' interaction aspects led to the development of the classification categories, i.e., interaction type, VR motion type and VR interaction space. Consequently, the documented VR locomotion techniques were assigned to the classification categories, creating four distinct VR locomotion types, which are visualized in Figure 3:

- Motion-based: The VR locomotion techniques under this type utilize some kind of physical movement to enable interaction, while supporting continuous motion in open VR spaces. This VR locomotion type includes such techniques as walking-in-place, redirected walking, arm swinging, gesture-based locomotion and reorientation.

- Room scale-based: This VR locomotion type utilizes physical movement to enable interaction, and it supports continuous motion (as with the motion-based type); however, the interaction takes place in VR environments whose size is limited by the real environment's size. The nomenclature for this locomotion type comes from the room-scale VR technology, which presents these interaction features [67]. The real-walking locomotion technique falls under this type.

- Controller-based: For this VR locomotion type, controllers are utilized to move the user artificially in the VR environment. The VR interaction space is open, and the motion is continuous. This type includes such techniques as joystick-based, human joystick, chair-based and head-directed locomotion.

- Teleportation-based: The VR locomotion techniques under this type utilize artificial interactions in open VR spaces with non-continuous movement, as the user's virtual viewpoint is instantaneously teleported to a predefined position by utilizing visual "jumps". Point and teleport is a VR locomotion technique that falls under this type.

The proposed typology manages to present four distinct types of VR locomotion. Motion-based locomotion differs from room scale-based in terms of the VR interaction space, while controller-based locomotion differs from teleportation-based in terms of the VR motion type. Motion-based and room scale-based locomotion differ from controller-based and teleportation-based locomotion in terms of their interaction type. Furthermore, the analysis of the reviewed VR locomotion techniques showed that techniques with physical interaction presented solely continuous VR motion, while artificial techniques were exclusively facilitating navigation in open, unlimited VR environments. Naturally, this feature of the proposed typology could be revised in future versions of the review, in case a new or updated VR locomotion technique does not fit under the extracted typology categories. 


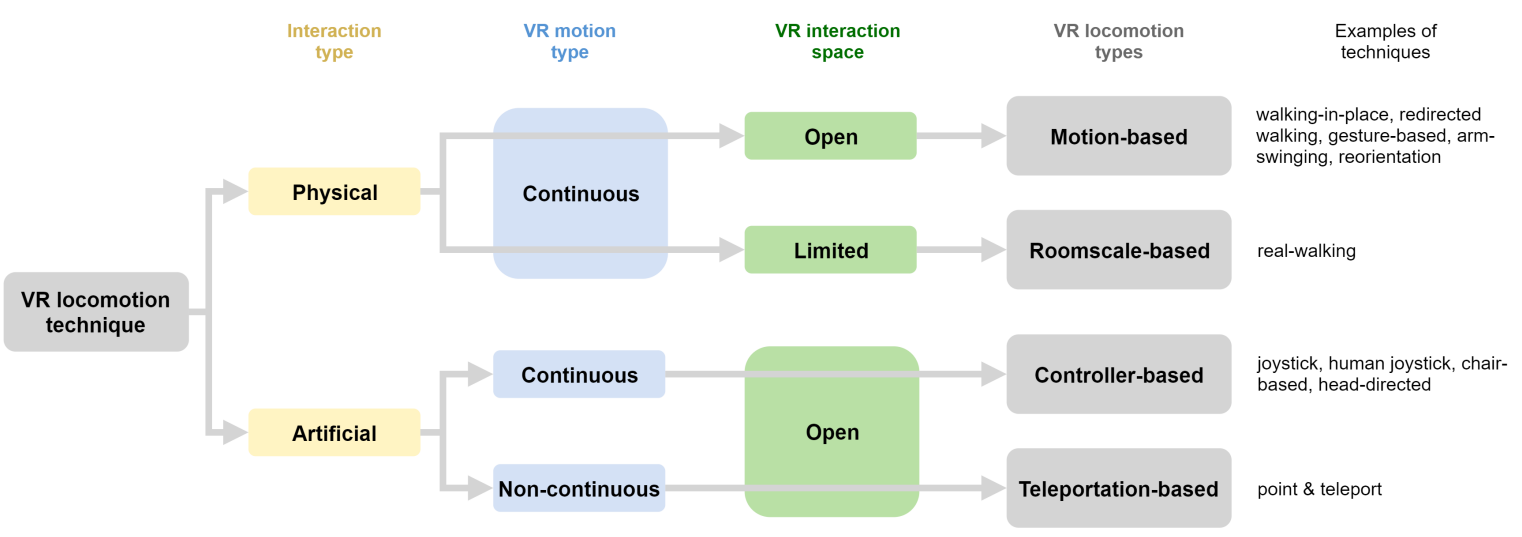

Figure 3. The VR locomotion typology.

The proposed typology can be a useful tool for researchers and users who want to present and describe the features of a VR locomotion technique utilizing a standardized description that clearly distinguishes one technique from another. The fact that the typology originates from the reviewed and studied locomotion techniques of the last three years provides an up-to-date character to the proposed VR locomotion types. These types can serve as a common ground for researchers of HCI and VR and the public who uses these systems to communicate the interaction aspects and functionalities that were previously difficult to describe and classify, thus enhancing the field's social impact. At the same time, the proposed VR locomotion typology, along with the systematic literature review, can affect positively the problem-solving capacity of HCI research in the VR field. Both works constitute a part of the conceptual work that has been highly needed in the HCI-in-VR field since the VR revival, addressing the organization of existing knowledge and the creation of concepts that can facilitate communication between research hypotheses and constructive work, i.e., designs [3].

\subsection{Study Limitations}

The diverse nature of the various VR locomotion techniques and their accompanying empirical studies presented challenges, leading to a series of compromises and assumptions that could also be perceived as limitations of this literature review.

First, a VR locomotion technique can integrate two or more locomotion techniques to facilitate navigation. For instance, point and teleport [12] utilizes gesture-based interaction to point to where the user wants to go, and the main motion takes place through teleportation. Naturally, these kinds of integrations include a dominant interaction metaphor. In this review, the VR locomotion techniques that integrate elements from other techniques were analyzed based on their dominant interaction aspects. In the aforementioned example, point and teleport was categorized as a teleportation-based technique, despite its gesture-based interaction aspects.

A second compromise has to do with the analysis of research topics. The categorization of a study as a usability, user experience, user perception or technical study took place following a Boolean logic, without examining the extent to which those topics were covered. For instance, even if only one aspect of the user experience was examined in a study (e.g., the sense of a user's VR presence [46]) along with a series of usability issues, the study was registered as having both a user experience and usability research topic. Naturally, it would be challenging, if not impossible or invalid, to quantify the extent to which research topics are covered. However, the followed Boolean approach might be considered a limitation of this systematic literature review.

Another limitation has to do with the normalization process that had to take place when shaping the research topic themes. The topics stand at a high description level, covering the research from a wide perspective, i.e., whether it is usability, user experience, etc. The main reason for that is that during the analysis, the majority of studies were focusing on general usability or several forms 
of the user experience. Even if some studies where addressing specific research topics, acting at a lower, more-focused level (e.g., reducing the unintended positional drift during walking-in-place locomotion [35,37]), the fact that many more studies were assessing topics at a higher level (e.g., [42]) led the review analysis of research topics to be performed at a normalized, high level, so that it covers all studies equally.

Moreover, the results of the reviewed, empirical studies were not included in the review as part of the research topics. The logic behind this decision was that the study results would completely move the shift from the VR locomotion techniques and their interaction aspects to the studies themselves and their characteristics (sample size, methodology, study design, etc.), while it would require a much more complex normalization process so that all results are comparable. Undoubtedly, a future extension of the review can move in that direction and extensively investigate the quality of research in the field.

Finally, the database query of the review is based on a predefined set of search terms. The defined search strategy conforms to the established procedures for systematic literature reviews [26], breaking down and addressing the research questions, while ensuring the reproducibility of the search. However, with VR being a dynamic technical and research field, predefined sets of search terms might not be able to cover the number of works that utilize new or unestablished terminology.

\section{Conclusions}

The presented systematic literature review shed more light on the new era of VR locomotion, analyzing state-of-the-art VR locomotion techniques and their empirical studies. Results showed that after the VR revival milestone, the focus of VR locomotion research is on physical interaction for navigating open VR environments with continuous motion, which is a format that can be further utilized in future studies or as a starting point for addressing and researching the field's less-researched areas, e.g., exploring artificial, teleportation-based techniques. Moreover, on the research side, the literature review also revealed the need for more user-centric, empirical research approaches, potentially under comparative settings.

Overall, the presented literature review provides researchers and developers with much interaction-related information regarding the studied VR locomotion techniques, so they are able to base their future designs on solid theoretical knowledge. This work, apart from organizing the field and documenting the research around this significant VR-related topic, synthesizes new conceptual knowledge, i.e., the proposed typology of VR locomotion techniques, which can be of use for researchers and users, acting as a keystone on which interested parties can build using their opinions and contributions.

Future work will address the quality of the research in the VR locomotion field, focusing further on the studies' characteristics (as stated in Section 4.4). Furthermore, the proposed typology will be updated based on future advancements, always aiming at making VR locomotion research accessible and useful to researchers and users.

Acknowledgments: I would like to thank Dimitra Chasanidou (Department of Software and Service Innovation, SINTEF Digital) for assisting with the literature review, being the independent expert, and also for providing valuable feedback on the examined topics. This research is funded by the Norwegian Research Council through the Centre for Service Innovation.

Conflicts of Interest: The author declares no conflict of interest. 


\section{References}

1. Olszewski, K.; Lim, J.J.; Saito, S.; Li, H. High-fidelity facial and speech animation for VR HMDs. ACM Trans. Graph. 2016, 35, 221.

2. Sun, H.M.; Li, S.P.; Zhu, Y.Q.; Hsiao, B. The effect of user's perceived presence and promotion focus on usability for interacting in virtual environments. Appl. Ergon. 2015, 50, 126-132.

3. Boletsis, C.; Cedergren, J.E.; Kongsvik, S. HCI research in Virtual Reality: A discussion of problem-solving. In Proceedings of the 11th International Conference on Interfaces and Human Computer Interaction, Lisbon, Portugal, 21-23 July 2017; pp. 263-267.

4. Hilfert, T.; König, M. Low-cost virtual reality environment for engineering and construction. Vis. Eng. 2016, $4,2$.

5. Giuseppe, R.; Wiederhold, B.K. The new dawn of virtual reality in health care: Medical simulation and experiential interface. Stud. Health Technol. Inform. 2015, 219, 3-6.

6. Kim, A.; Darakjian, N.; Finley, J.M. Walking in fully immersive virtual environments: An evaluation of potential adverse effects in older adults and individuals with Parkinson's disease. J. Neuroeng. Rehabilit. 2017, 14, 16.

7. Moreira, P.; de Oliveira, E.C.; Tori, R. Impact of Immersive Technology Applied in Computer Graphics Learning. In Proceedings of the Brazilian Symposium on Computers in Education, Uberlandia, MG, Brazil, 24-27 October 2016; pp. 410-419.

8. Reinert, B.; Kopf, J.; Ritschel, T.; Cuervo, E.; Chu, D.; Seidel, H.P. Proxy-guided Image-based Rendering for Mobile Devices. Comput. Graph. Forum 2016, 35, 353-362.

9. Wang, H.; Mahmud, M.S.; Fang, H.; Wang, C. Wireless Health; Springer: Berlin, Germany, 2016.

10. Smith, P.A. Using Commercial Virtual Reality Games to Prototype Serious Games and Applications. In Proceedings of the 9th International Conference on Virtual, Augmented and Mixed Reality, Vancouver, BC, Canada, 9-14 July 2017; Springer: Berlin, Germany, 2017; pp. 359-368.

11. Zhang, X.; Gračanin, D.; Matković, K. Using Linked Data for Interactive 3D Web Content Integration. In Proceedings of the 19th International ACM Conference on 3D Web Technologies, Vancouver, BC, Canada, 8-10 August 2014; ACM: New York, NY, USA, 2014; p. 147.

12. Bozgeyikli, E.; Raij, A.; Katkoori, S.; Dubey, R. Point \& teleport locomotion technique for virtual reality. In Proceedings of the Annual Symposium on Computer-Human Interaction in Play, Austin, TX, USA, 16-19 October 2016; ACM: New York, NY, USA, 2016; pp. 205-216.

13. Hale, K.S.; Stanney, K.M. Handbook of Virtual Environments: Design, Implementation, and Applications; CRC Press: Boca Raton, FL, USA, 2014.

14. Bowman, D.; Kruijff, E.; LaViola, J.J., Jr.; Poupyrev, I.P. 3D User Interfaces: Theory and Practice, CourseSmart eTextbook; Addison-Wesley: Boston, MA, USA, 2004.

15. Bowman, D.A.; Koller, D.; Hodges, L.F. Travel in immersive virtual environments: An evaluation of viewpoint motion control techniques. In Proceedings of the IEEE Virtual Reality Annual International Symposium, Albuquerque, NM, USA, 1-5 March 1997; IEEE: Piscataway, NJ, USA, 1997; pp. 45-52.

16. Bowman, D.A.; Koller, D.; Hodges, L.F. A methodology for the evaluation of travel techniques for immersive virtual environments. Virtual Real. 1998, 3, 120-131.

17. Arns, L.L. A New Taxonomy for Locomotion in Virtual Environments; Iowa State University: Ames, IA, USA, 2002.

18. Ferracani, A.; Pezzatini, D.; Bianchini, J.; Biscini, G.; Del Bimbo, A. Locomotion by Natural Gestures for Immersive Virtual Environments. In Proceedings of the 1st International Workshop on Multimedia Alternate Realities, Amsterdam, The Netherlands, 16 October 2016; ACM: New York, NY, USA, 2016; pp. 21-24.

19. Wilson, P.T.; Kalescky, W.; MacLaughlin, A.; Williams, B. VR locomotion: Walking > walking in place $>$ arm swinging. In Proceedings of the ACM SIGGRAPH Conference on Virtual-Reality Continuum and Its Applications in Industry-Volume 1, Zhuhai, China, 3-4 December 2016; ACM: New York, NY, USA, 2016; pp. 243-249.

20. Fernandes, A.S.; Feiner, S.K. Combating VR sickness through subtle dynamic field-of-view modification. In Proceedings of the IEEE Symposium on 3D User Interfaces, Greenville, SC, USA, 19-20 March 2016; IEEE: Piscataway, NJ, USA, 2016; pp. 201-210. 
21. Ward, M.; Azuma, R.; Bennett, R.; Gottschalk, S.; Fuchs, H. A demonstrated optical tracker with scalable work area for head-mounted display systems. In Proceedings of the 1992 Symposium on Interactive 3D Graphics, San Diego, CA, USA, 10-11 December 1990; ACM: New York, NY, USA, 1990; pp. 43-52.

22. Bruder, G.; Steinicke, F. Threefolded Motion Perception During Immersive Walkthroughs. In Proceedings of the ACM Symposium on Virtual Reality Software and Technology, Edinburgh, UK, 11-13 November 2014; ACM: New York, NY, USA, 2014; pp. 177-185.

23. Bozgeyikli, E.; Bozgeyikli, L.; Raij, A.; Katkoori, S.; Alqasemi, R.; Dubey, R. Virtual Reality Interaction Techniques for Individuals with Autism Spectrum Disorder: Design Considerations and Preliminary Results. In Proceedings of the International Conference on Human-Computer Interaction, Toronto, ON, Canada, 17-22 July 2016; Springer: Berlin, Germany, 2016; pp. 127-137.

24. Reddit-List of VR Locomotion Methods. Available online: https://www.reddit.com/r/Vive/wiki/ locomotion_methods (accessed on 29 July 2017).

25. Reddit-What is Your Favourite Form of Locomotion in VR? Available online: https://www.reddit.com/r/ Vive/comments /4rj357/what_is_your_favourite_form_of_locomotion_in_vr/ (accessed on 29 July 2017).

26. Kitchenham, B. Procedures for Performing Systematic Reviews; Technical Report TR/SE-0401; Keele University: Newcastle upon Tyne, UK; National ICT Australia Ltd.: Sydney, Australia, 2004.

27. Brereton, P.; Kitchenham, B.A.; Budgen, D.; Turner, M.; Khalil, M. Lessons from applying the systematic literature review process within the software engineering domain. J. Syst. Softw. 2007, 80, 571-583.

28. Beecham, S.; Baddoo, N.; Hall, T.; Robinson, H.; Sharp, H. Motivation in Software Engineering: A systematic literature review. Inf. Softw. Technol. 2008, 50, 860-878.

29. Falagas, M.E.; Pitsouni, E.I.; Malietzis, G.A.; Pappas, G. Comparison of PubMed, Scopus, Web of Science, and Google Scholar: strengths and weaknesses. FASEB J. 2008, 22, 338-342.

30. Jacso, P. As we may search-comparison of major features of the Web of Science, Scopus, and Google Scholar citation-based and citation-enhanced databases. Curr. Sci. 2005, 89, 1537-1547.

31. Vom Brocke, J.; Simons, A.; Niehaves, B.; Riemer, K.; Plattfaut, R.; Cleven, A. Reconstructing the giant: On the importance of rigour in documenting the literature search process. In Proceedings of the European Conference on Information Systems, Verona, Italy, 8-10 June 2009; pp. 2206-2217.

32. Webster, J.; Watson, R.T. Analyzing the past to prepare for the future: Writing a literature review. MIS $Q$. 2002, 26, 13-23.

33. Grechkin, T.Y.; Plumert, J.M.; Kearney, J.K. Dynamic affordances in embodied interactive systems: The role of display and mode of locomotion. IEEE Trans. Vis. Comput. Graph. 2014, 20, 596-605.

34. Skopp, N.A.; Smolenski, D.J.; Metzger-Abamukong, M.J.; Rizzo, A.A.; Reger, G.M. A Pilot Study of the VirtuSphere as a Virtual Reality Enhancement. Int. J. Hum.-Comput. Interact. 2014, 30, $24-31$.

35. Nilsson, N.C.; Serafin, S.; Nordahl, R. A comparison of different methods for reducing the unintended positional drift accompanying walking-in-place locomotion. In Proceedings of the IEEE Symposium on 3D User Interfaces, Minneapolis, MN, USA, 29-30 March 2014; IEEE: Piscataway, NJ, USA, 2014; pp. 103-110.

36. Nilsson, N.C.; Serafin, S.; Nordahl, R. Establishing the range of perceptually natural visual walking speeds for virtual walking-in-place locomotion. IEEE Trans. Vis. Comput. Graph. 2014, 20, 569-578.

37. Nilsson, N.C.; Serafin, S.; Nordahl, R. A comparison of four different approaches to reducing unintended positional drift during walking-In-Place locomotion. In Proceedings of the IEEE Virtual Reality, Minneapolis, MN, USA, 29 March-2 April 2014; IEEE: Piscataway, NJ, USA, 2014; pp. 1-2.

38. Caggianese, G.; Gallo, L.; Neroni, P. Design and preliminary evaluation of free-hand travel techniques for wearable immersive virtual reality systems with egocentric sensing. In Proceedings of the International Conference on Augmented and Virtual Reality, Lecce, Italy, 31 August-3 September 2015; Springer: Berlin, Germany, 2015; pp. 399-408.

39. Harris, A.; Nguyen, K.; Wilson, P.T.; Jackoski, M.; Williams, B. Human joystick: Wii-leaning to translate in large virtual environments. In Proceedings of the ACM SIGGRAPH International Conference on Virtual-Reality Continuum and its Applications in Industry, Shenzhen, China, 30 November-2 December 2014; ACM: New York, NY, USA, 2014; pp. 231-234.

40. Nescher, T.; Huang, Y.Y.; Kunz, A. Planning redirection techniques for optimal free walking experience using model predictive control. In Proceedings of the IEEE Symposium on 3D User Interfaces, Minneapolis, MN, USA, 29-30 March 2014; IEEE: Piscataway, NJ, USA, 2014; pp. 111-118. 
41. Nabiyouni, M.; Scerbo, S.; DeVito, V.; Smolen, S.; Starrin, P.; Bowman, D.A. Design and evaluation of a visual acclimation aid for a semi-natural locomotion device. In Proceedings of the IEEE Symposium on 3D User Interfaces, Arles, France, 23-24 March 2015; IEEE: Piscataway, NJ, USA, 2015; pp. 11-14.

42. Nabiyouni, M.; Saktheeswaran, A.; Bowman, D.A.; Karanth, A. Comparing the performance of natural, semi-natural, and non-natural locomotion techniques in virtual reality. In Proceedings of the IEEE Symposium on 3D User Interfaces, Arles, France, 23-24 March 2015; IEEE: Piscataway, NJ, USA, 2015; pp. 3-10.

43. Bruder, G.; Lubas, P.; Steinicke, F. Cognitive resource demands of redirected walking. IEEE Trans. Vis. Comput. Graph. 2015, 21, 539-544.

44. Schmidt, D.; Kovacs, R.; Mehta, V.; Umapathi, U.; Köhler, S.; Cheng, L.P.; Baudisch, P. Level-ups: Motorized stilts that simulate stair steps in virtual reality. In Proceedings of the 33rd Annual ACM Conference on Human Factors in Computing Systems, Seoul, Korea, 18-23 April 2015; ACM: New York, NY, USA, 2015; pp. 2157-2160.

45. Kruijff, E.; Riecke, B.; Trekowski, C.; Kitson, A. Upper body leaning can affect forward self-motion perception in virtual environments. In Proceedings of the 3rd ACM Symposium on Spatial User Interaction, Los Angeles, CA, USA, 8-9 August 2015; ACM: New York, NY, USA, 2015; pp. 103-112.

46. De la Rubia, E.; Diaz-Estrella, A. Natural locomotion based on foot-mounted inertial sensors in a wireless virtual reality system. Presence Teleoper. Virtual Environ. 2015, 24, 298-321.

47. Zank, M.; Kunz, A. Using locomotion models for estimating walking targets in immersive virtual environments. In Proceedings of the International Conference on Cyberworlds, Visby, Sweden, 7-9 October 2015; IEEE: Piscataway, NJ, USA, 2015; pp. 229-236.

48. Langbehn, E.; Eichler, T.; Ghose, S.; von Luck, K.; Bruder, G.; Steinicke, F. Evaluation of an omnidirectional walking-in-place user interface with virtual locomotion speed scaled by forward leaning angle. In Proceedings of the GI Workshop on Virtual and Augmented Reality, Sankt Augustin, Germany, 10-11 September 2015; pp. 149-160.

49. McCullough, M.; Xu, H.; Michelson, J.; Jackoski, M.; Pease, W.; Cobb, W.; Kalescky, W.; Ladd, J.; Williams, B. Myo arm: Swinging to explore a VE. In Proceedings of the ACM SIGGRAPH Symposium on Applied Perception, Tübingen, Germany, 13-14 September 2015; ACM: New York, NY, USA, 2015; pp. 107-113.

50. Borrego, A.; Latorre, J.; Llorens, R.; Alcañiz, M.; Noé, E. Feasibility of a walking virtual reality system for rehabilitation: objective and subjective parameters. J. Neuroeng. Rehabilit. 2016, 13, 68.

51. Zank, M.; Kunz, A. Eye tracking for locomotion prediction in redirected walking. In Proceedings of the IEEE Symposium on 3D User Interfaces, Greenville, SC, USA, 19-20 March 2016; IEEE: Piscataway, NJ, USA, 2016; pp. 49-58.

52. Tregillus, S.; Folmer, E. Vr-step: Walking-in-place using inertial sensing for hands free navigation in mobile vr environments. In Proceedings of the CHI Conference on Human Factors in Computing Systems, San Jose, CA, USA, 7-12 May 2016; ACM: New York, NY, USA, 2016; pp. 1250-1255.

53. Sun, Q.; Wei, L.Y.; Kaufman, A. Mapping virtual and physical reality. ACM Trans. Graph. (TOG) 2016, 35, doi:10.1145/2897824.2925883.

54. Bozgeyikli, E.; Raij, A.; Katkoori, S.; Dubey, R. Locomotion in Virtual Reality for Individuals with Autism Spectrum Disorder. In Proceedings of the Symposium on Spatial User Interaction, Tokyo, Japan, 15-16 Ocotober 2016; ACM: New York, NY, USA, 2016; pp. 33-42.

55. Kruijff, E.; Marquardt, A.; Trepkowski, C.; Lindeman, R.W.; Hinkenjann, A.; Maiero, J.; Riecke, B.E. On Your Feet!: Enhancing Vection in Leaning-Based Interfaces through Multisensory Stimuli. In Proceedings of the 2016 Symposium on Spatial User Interaction, Tokyo, Japan, 15-16 Ocotober 2016; ACM: New York, NY, USA, 2016; pp. 149-158.

56. Nishi, A.; Hoshino, K.; Kajimoto, H. Straightening Walking Path Using Redirected Walking Technique. In Proceedings of the ACM SIGGRAPH, Anaheim, CA, USA, 24-28 July 2016; ACM: New York, NY, USA, 2016; p. 61.

57. Argelaguet, F; Maignant, M. GiAnt: stereoscopic-compliant multi-scale navigation in VEs. In Proceedings of the ACM Conference on Virtual Reality Software and Technology, Munich, Germany, 2-4 November 2016; ACM: New York, NY, USA, 2016; pp. 269-277.

58. Cardoso, J. Comparison of gesture, gamepad, and gaze-based locomotion for VR worlds. In Proceedings of the ACM Conference on Virtual Reality Software and Technology, Munich, Germany, 2-4 November 2016; ACM: New York, NY, USA, 2016; pp. 319-320. 
59. Paris, R.A.; McNamara, T.P.; Rieser, J.J.; Bodenheimer, B. A comparison of methods for navigation and wayfinding in large virtual environments using walking. In Proceedings of the IEEE Virtual Reality, Los Angeles, CA, USA, 18-22 March 2017; IEEE: Piscataway, NJ, USA, 2017; pp. 261-262.

60. Xu, M.; Murcia-López, M.; Steed, A. Object location memory error in virtual and real environments. In Proceedings of the IEEE Virtual Reality, Los Angeles, CA, USA, 18-22 March 2017; IEEE: Piscataway, NJ, USA, 2017; pp. 315-316.

61. Fisher, J.A.; Garg, A.; Singh, K.P.; Wang, W. Designing intentional impossible spaces in virtual reality narratives: A case study. In Proceedings of the IEEE Virtual Reality, Los Angeles, CA, USA, 18-22 March 2017; IEEE: Piscataway, NJ, USA, 2017; pp. 379-380.

62. Kitson, A.; Hashemian, A.M.; Stepanova, E.R.; Kruijff, E.; Riecke, B.E. Comparing leaning-based motion cueing interfaces for virtual reality locomotion. In Proceedings of the IEEE Symposium on 3D User Interfaces, Los Angeles, CA, USA, 18-19 March 2017; IEEE: Piscataway, NJ, USA, 2017; pp. 73-82.

63. Kim, J.S.; Gracanin, D.; Matkovic, K.; Quek, F.K. The Effects of Finger-Walking in Place (FWIP) for Spatial Knowledge Acquisition in Virtual Environments. Lect. Notes Comput. Sci. 2010, 6133, 56-67.

64. Wright, P.; Blythe, M. User experience research as an inter-discipline: Towards a UX Manifesto. In Proceedings of the BHCI '07 Workshop on Towards a UX Manifesto, Lancaster, UK, 3-7 September 2007; pp. 65-70.

65. Bargas-Avila, J.A.; Hornbæk, K. Old Wine in New Bottles or Novel Challenges: A Critical Analysis of Empirical Studies of User Experience. In Proceedings of the SIGCHI Conference on Human Factors in Computing Systems, Vancouver, BC, Canada, 7-12 May 2011; ACM: New York, NY, USA, 2011; pp. 2689-2698.

66. Van Veen, H.A.; Distler, H.K.; Braun, S.J.; Bülthoff, H.H. Navigating through a virtual city: Using virtual reality technology to study human action and perception. Futur. Gener. Comput. Syst. 1998, 14, $231-242$.

67. Langbehn, E.; Lubos, P.; Bruder, G.; Steinicke, F. Application of redirected walking in room-scale VR. In Proceedings of the IEEE Virtual Reality (VR), Los Angeles, CA, USA, 18-22 March 2017; IEEE: Piscataway, NJ, USA, 2017; pp. 449-450.

(C) 2017 by the author. Licensee MDPI, Basel, Switzerland. This article is an open access article distributed under the terms and conditions of the Creative Commons Attribution (CC BY) license (http:/ / creativecommons.org/licenses/by/4.0/). 